\title{
Peripatetic electronic teachers in higher education
}

\author{
David Squires \\ School of Education, King's College London, email: david.squires@kcl.ac.uk
}

This paper explores the idea of information and communications technology providing a medium enabling higher education teachers to act as freelance agents. The notion of a 'Peripatetic Electronic Teacher' (PET) is introduced to encapsulate this idea. PETs would exist as multiple telepresences (pedagogical, professional, managerial and commercial) in PET-worlds; global networked environments which support advanced multimedia features. The central defining rationale of a pedagogical presence is described in detail and some implications for the adoption of the PET-world paradigm are discussed. The ideas described in this paper were developed by the author during a recently completed Short-Term British Telecom Research Fellowship, based at the BT Adastral Park.

\section{Introduction}

Most people would agree that the role of teachers in higher education will be changed by the advent of modern information and communication technology (ICT). At the very least open and distant learning (ODL) will become an established feature of educational provision rather than a specialist aspect. This is already evident in the increasing use of ODL in combination with conventional forms of course delivery by universities. More radically it is claimed that there will be a fundamental change in the nature of educational institutions, leading to the notion of virtual university, e.g. the Western Governors University (http://www.wgu.ed). These claims have in common the notion of utilizing the distributed nature of ICT to provide partnerships between teachers and learners that are not defined by either spatial or temporal constraints.

While the concept of a virtual university is radical, there is an implicit assumption that educators will still be primarily affiliated to one institution. An even more radical claim is that ICT will break exclusive links between educators and a single institution. Just as future learners may be seen as clients contracting to receive educational provision from a range of providers, educators may be seen as independent workers offering their services to learners on 
demand. These educators will not be confined to the classroom, rather they will be electronic workers providing a virtual presence in public spaces, the workplace and the home. A new type of peripatetic electronic teacher (PET) will emerge. Aristotle, the first peripatetic teacher, used to walk around the Lyceum as he taught; PETs will surf around the Net as they teach.

These changes were foreseen in principle long before the advent of the Web. In 1970 Ivan Illich (1971) introduced the idea of a 'learning web' providing global access to learning resources and enabling free communication between learners and teachers. A combination of the idea of a learning web and the possibilities afforded by ICT leads to the notion of a peripatetic electronic teacher. PETs will act as information brokers, providing routes for learners to search networked information systems. They will provide virtual asynchronous tutorials for small groups and individuals; act as the teacher in a virtual classroom environment, manage synchronous virtual seminars, and moderate discussion lists/bulletin boards. On occasion they will provide face-to-face teaching sessions, which will be advertised on the Web. Instead of using 'off-the-shelf' curriculum materials PETs will use multimedia authoring systems to design and publish materials, either for general circulation or as bespoke products developed in response to client demand.

Initial development work on a design specification and prototype for a PET software environment has been completed through a BT Short-Term Research Fellowship based at the BT Adastral Park. This paper describes this design specification.

\section{A PET design rationale}

Squires (1999) describes the original design rationale for a PET as an 'Assistant' providing a seamless interface to a diverse range of design, navigation and communication tools:

- A peripatetic network-based teacher will need to maintain an overt presence on the Web. At a practical level this involves being able to handle diverse requests from clients, advertise services and communicate with colleagues.

- Some teachers may wish to combine a Web-based virtual presence with contract teaching commitments in diverse locations. These teachers will need to be contactable in a variety of locations.

- Brokering information will require access to state of the art network navigation systems, and possibly the development of navigation tools customized to the needs of education. Navigation tools with different approaches will need to be presented in a coherent and synergistic way.

- Designing learning materials will require access to a range of complementary authoring environments.

- Digital publishing will need support - copyright will need to be protected, payments will need to be made and links with software publishing agencies will need to be maintained.

This is a utilitarian and functional view which originates from a view of the Internet/Web as primarily an information and distribution mechanism. However, the 'superhighway' metaphor is immature - it is being replaced by the notion of global networks as social places. Schlager, Fusco and Schank (in press) cite McFarlane (1996) in support of this emerging notion; they 
regard a networked environment as a gathering place, or agora, that 'brings people together, encourages participation, and supports creativity, a place that is always growing, adapting, and changing in response to new ideas and initiatives'. This leads to a rather different perception of the design of a PET Assistant. It is not a well-ordered and comprehensive toolbox that is needed, but rather a flexible virtual space/environment which allows a PET to have an effective and functional telepresence on the Web. In this sense it is a question of designing a PET-World, inhabited by PETs and learners, rather than a PET Assistant.

With this perspective in mind a design specification for a PET-World has been developed based on the idea of four presence domains:

- Pedagogical presence where the PET appears as a teacher, playing roles such as instructor, coach, mentor, tutor, scaffolder and expert.

- Professional presence where the PET appears as a member of the teaching profession, playing roles such as colleague, committee member, trainer and trainee.

- Commercial presence where the PET appears as a freelance worker available for hire, playing roles such as consultant, personal tutor and publisher.

- Managerial presence where the PET appears as an administrator, scheduling teaching commitments, validating learners attendance/achievements and managing course enrolment.

All domains are important in realizing a holistic perception of the role and scope of a PET-World, and initial design specifications exist for each domain. As PET-Worlds are developed other presences may be required. However, the pedagogical domain provides a central defining rationale and the specification for this domain has been developed to the greatest extent. The remainder of this paper will describe this specification and reflect on the implications of implementing the design.

\section{Pedagogical telepresence}

Teachers communicate with learners through two fundamental channels: exposition and discourse. Pedagogical presence is defined by the character of exposition to learners and the nature of discourse with learners. From a constructivist perspective exposition is not simply a question of presenting ideas, facts and so on for consumption, i.e. pedagogical presence is not simply presentational. Rather it is a question of exposing learners to learning situations which encourage them to develop ideas and concepts in ways which match their idiosyncratic needs and demands. Thus exposition will be a mix of presenting ideas and concepts for consideration, setting problems to be solved and organizing projects. An essential aspect of good teaching is feedback and advice based on effective assessment. Discourse provides a way of giving this feedback, with the teacher assuming roles such as guide, mentor, adviser, moderator, critic, leader and instigator in discussions both between teacher and learner(s) and between learners (peer group collaboration). In this sense discourse provides opportunities for the teacher to scaffold learners' activities.

Exposition and discourse will remain as defining communication channels for PETs, but the nature and scope of these channels will change, e.g asynchronous conferencing 


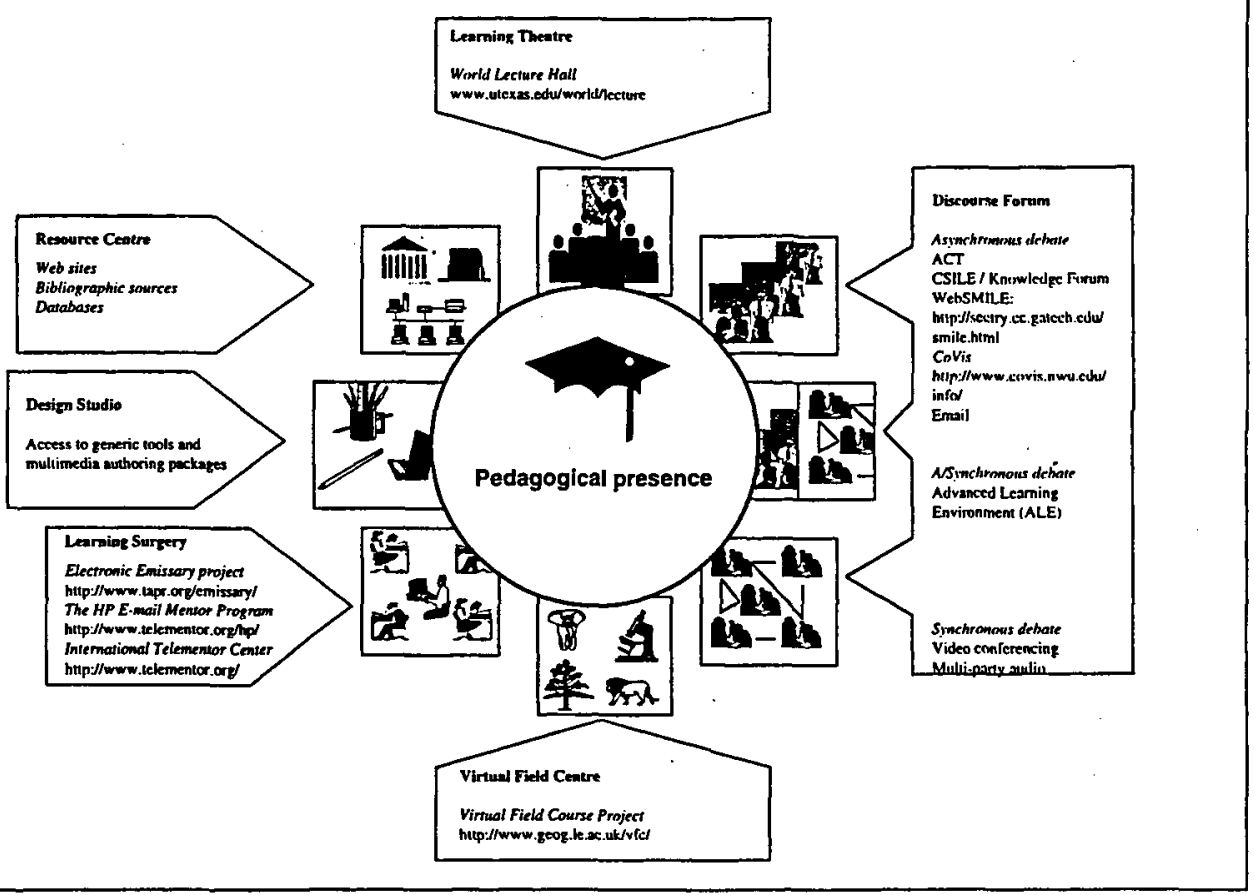

Figure 1

systems are introducing new aspects of discourse between teacher and learner. A consideration of exposition and discourse in a PET-World context suggests a number of 'learning rooms' within the Pedagogical Presence Domain (see Figure 1):

- a Learning Theatre in which exposition can take place in an interactive distributed multimedia environment;

- a Discourse Forum based on access to a range of synchronous, asynchronous and hybrid a/synchronous systems providing opportunities for the PET to scaffold learners' activities;

- a Virtual Field Centre in which the PET acts as guide and designer of thematically constructed explorations of the Web;

- a Learning Surgery in which a PET can assume the roles of tutor and mentor to respond to requests for specific help and advice form individuals and small groups;

- a Design Studio providing a PET with access to a range of design tools to develop curriculum materials;

- a Resource Centre where a PET is a guide to the selection and retrieval of information.

\section{Learning theatre}

Providing access to diverse, extensive and high-quality materials to support networked lecture presentations is an immediately obvious rationale. Didactic presentations of 
knowledge can be augmented by use of slides, video, animations and simulations - very much as in an Open University television programme. Basically this rationale rests on the idea of a (multimedia) replication of conventional lecture presentations.

This is a limited view which does not take account of a constructivist learner-centred focus. Norman (1998), in describing the use of multimedia networked classrooms at the University of Maryland, observes that the trend has been to "evolve from presentations to explorations, from passive learning to active engagement and from "sage on the stage" to "guide on the side" ' (p. 42). Norman cites Shneiderman, Norman and Borkowski (1995) as emphasizing this trend in stating that the electronic classrooms at the university were originally called 'teaching theatres', but with experience were renamed 'learning theatres'. This term is borrowed to describe an area within the Pedagogical Presence Domain.

A learning theatre will combine the possibilities of hi-fidelity presentation with synchronous interaction, leading to the notion of participative exposition. Learning theatres will be examples of shared spaces as described by Bradley, Walker and McGrath (1996). The inhabitants of these spaces will be the teacher(s) and the learners. Interaction will typically be orchestrated by the teacher, but there will be possibilities for learnerlearner interaction as well. Emerging virtual reality technologies provide exciting possibilities in this context, e.g. systems such as The Mirror have a three-dimensional shared space (Walker, 1997) which combines multiple avatar presence with synchronous text-based discourse. Future worlds may include multi-party audio. It is not hard to imagine a learning theatre populated by avatars (teacher(s) and learners) with real time audio conferencing.

\section{Identity}

A conventional classroom provides teachers with a social space in which they can project their personality and exercise their role as a teacher, i.e. a forum for maintaining a pedagogical presence. Learning theatres will need to afford PETs similar possibilities. Personal appearance may offer one way of providing a personality affordance. For example, 'The Mirror included a choice of four avatars with clothing which could be coloured from an extensive palette' (Walker, 1997, p.32). A similar approach could be adopted in learning theatres, with PETs offered the facilities to design their own appearance and, if multi-party audio is used, their own voice characteristics. If synchronous text based discourse is employed, there may be some merit in presenting text contributions as characteristic handwriting rather than as typed script. Teachers and learners could choose a handwriting style from a style library. As they typed in text it could automatically be converted into script presented in this style. Another possibility is the availability of a voice to text system for use by the PET.

\section{Learning theatre management}

Well run classrooms are characterized by diverse practice: whole group presentation, small group work, individual work and circus activities. Learning theatres will need to offer PETs similar management affordance. The use of 'auras' (grouping algorithms used to produce scaleable worlds) could be relevant in this context. These algorithms have been developed to make shared spaces populated by large numbers of avatars technically feasible and practically manageable by users. Users are placed in need-to-know groups, enabling the system to control the amount of information sent to any user. The use of auras could also 
open up the possibility of massive participatory exposition, i.e. lessons in which large numbers of students could participate. These could be celebrity lessons given by 'excellent PETs'.

\section{Motivation}

Trials of The Mirror indicate 'the importance of special events and the pulling power of celebrities in maintaining the community' (Walker, 1997, p. 37). The notion of massive participatory exposition is relevant here. This raises issues of PETs working in teams. PETs would spend most of their time working with 'their' class, but would also act as an associate PET for special events. Of course, there are possibilities for team teaching as well.

\section{Authority}

What are the new forms of misbehaviour and ill-discipline that will arise in a virtual environment? Vandalism may take the form of deliberate malicious annotations to materials presented in open collaborative environments - multimedia graffiti. It may take the form of deleting and/or changing other peoples' work. Subtle changes may be the most insidious. Another form of ill-discipline may be unacceptable behaviour in open environments (e.g. MUDs) or the use of unacceptable language (e.g. in discussion groups). Will 'flaming' take on a new meaning? Hacking into confidential systems (e.g. learner records) may be a problem. Virtual discipline will raise a number of software development issues, e.g. providing controlled access to the Web, creating 'protected' user groups, and allowing censorship through blocking and regulatory systems (Martin, 1997).

\section{Discourse forum}

In conventional educational settings discourse is synchronous: classroom discussion, seminar groups, conversations between teachers and learners. While new technology opens up possibilities for replicating synchronous discourse (video conferencing, multi-party audio, telephone conferencing and text-based chat lines), perhaps the most interesting development is the emergence of asynchronous discourse systems. The fundamental claim here is that asynchronous discourse encourages reflection. Of course, the two forms can be combined to give a/synchronous discourse systems.

The notion of a discourse forum does not have a conventional equivalent, which attempts to provide a space defined in terms of a mixture of synchronous and asynchronous debate and exchange of information. A number of design issues have emerged in the design and development of systems such as those which would feature in a discourse forum: annotated discourse, configured discourse, and multimedia discourse.

\section{Annotated discourse}

It is commonly argued that asynchronous discussion fosters reflection leading to more thoughtful and considered responses. This begs the issue of how learners can adequately represent their reflective thinking. One common approach is to provide labelling facilities, e.g. as in the Asynchronous Conferencing Tool (ACT) described by Sloffer, Duebur and Duffy (1999). In ACT messages can be labelled as hypothesis, important point, evidence, and learning issue. In some systems there are reserved labels for teacher use only, e.g. a problem-setting label or a summary label. 
Configured discourse

Asynchronous discussions can also be recorded allowing participants to look at the history of a discussion. This in turn leads to attempts to represent discourse history. A variety of techniques exist. Discourse history can be represented in terms of argument threads, tasks or simply as a temporal record. Each of these approaches has its advantages and disadvantages.

Multimedia discourse

A number of systems, e.g. the Collaborative and Multimedia Interactive Learning Environment (Hübscher, Puntambekar and Guzdial, 1997), allow multimedia annotations. It is likely that the nature of discourse labels will change to encompass multimedia objects in addition to text: voice notes, video, graphics, pointers to URLs, etc.

\section{Virtual field centre}

Project-based activities involving information and data-gathering, analysis, synthesis and report are commonly advocated as a means of providing authentic learning experiences. Field-trip-based activities are a common manifestation of a project-based approach - visits to natural habitats to explore ecological issues, visits to museums, art galleries, visits to foreign covntries, etc. In a Web context it is possible to think of virtual field trips: guided explorations of natural habitats which would include simulations, visits to resource centre Web sites, and so on; art trips involving virtual visits to thematically selected exhibitions; historical trips involving visits to museums; and cultural trips to other countries with an emphasis on communication in the local language. The JISC-funded Virtual Field Course Project (http://www.geog.le.ac.uk/vfc), featuring collaboration between the University of Leicester, Birkbeck College, Oxford Brookes University and City University, provides an example of a development in this area.

In a virtual field trip the PET would have two roles: (i) designing and organizing the trip and (ii) acting as guide and navigator during the trip.

\section{Navigation design}

The design of the trip will involve the identification of relevant sites and proposing a route(s) for learners. Maps, signposts and itineraries all suggest themselves as navigational devices. However, as Platt and Willard (1998) point out, these techniques are not sensitive to temporal changes:

There are many techniques used in existing virtual environments that aim to reduce this risk of 'getting lost', such as signposts, maps, landmarks and viewpoints. However, these are generally set when the environment is originally designed and based on the designer's view of what is an interesting part of the world. Crossley et al. (1997) predict that 3D virtual environments will become more organic, being built and updated dynamically, with greater personalisation of the worlds and greater interactivity with objects. Therefore, designer-driven navigation aids may not be able to reflect such developments in the environment brought about by user interaction or changing information.

This is a serious design issue for virtual field trips. In these trips there should be possibilities for learners to explore by adapting/modifying the route(s) so as to foster serendipitous learning: they should not be constrained by the designer's original intentions. These adaptations in themselves provide a rich set of educational materials, implying that 
the virtual field trips should be mutable (Anderson and McGrath, 1997) to reflect changes in the range of suitable locations over time and the experiences of previous learners.

Platt and Willard (1998) have proposed three techniques that 'would be able to evolve along with the space and the community':

- The idea for virtual cairns is derived from the piles of stones that hill walkers place to mark routes and indicate points of interest. Platt and Willard describe virtual cairns as viewpoints that can be set by users rather than the designer. With a viewpoint there is an associated object or collection of objects, allowing users 'to see which positions or routes are important to other users, so the whole community contributes to the understanding of the space'. If the movement of users is monitored virtual cairns could be placed at frequently visited locations. It would be possible to link virtual cairns to other resources - video, text, etc. In a virtual field trip the temporal and spatial sensitivity of virtual cairns, the implicit collaboration between learners in adding and changing cairns, and the possibilities afforded by multimedia resource links to cairns make them a very attractive design technique.

- Trails correspond to the routes most frequently taken by users. These can be generated by automatically tracking the routes of users and synthesizing the most popular routes. They can be represented graphically in a virtual space. In a virtual field trip some default routes could be included to act as an introduction for learners.

- Intelligent tours are based on the idea of storing how people use a space and using this information to propose the most interesting route. The Information Garden (Crossley, Davies, Taylor-Hendry and McGrath, 1997) provides an example of an intelligent tour. Platt and Willard (1998) describe the simplest case of an intelligent tour as when 'the system might only be aware of the location of objects or information in the space, and move the user via these locations'. They propose that tracking other people in the environment and being aware of popular routes and locations, recent changes in objects and information, and the specific interests of users would make the system more useful. This would allow the creation of 'a tailor-made route for any particular user, ensuring that they discover any relevant parts of the environment (information or people) whilst moving quickly and effectively through the space' (Platt and Willard, 1998). Intelligent tours could open up the possibility of matching virtual field trips to the needs of individual learners.

\section{- Learning surgery}

A significant component of students' total learning experience is access to dedicated teaching support on a personal basis. In this context learners have a chance to express their own learning difficulties and get help geared to their own needs. Personal teaching in a conventional context is typically provided by a tutor working with individuals or small groups. The notion of mentoring, with subject experts offering individual learners advice and information, is also relevant in this context. There is a clear role for PETs to act as tutors, utilizing networked conferencing systems and email to provide synchronous and asynchronous support at a personal level. Tutoring by PETs leads to the idea of a Learning Surgery where individual learners can visit a PET for personal assistance. Conferencing systems also open up the possibility of putting learners in contact with expert mentors from universities, industry and commerce. 


\section{Individual and small group tutorials}

Synchronous communication, either between a PET and an individual learner or a small group of learners, offers possibilities of replicating many of the aspects of conventional personal and small group tutorials. Collaborative artefacts, such as interactive whiteboards and shared real-time documents and files, will be very important in the short- and mediumterm future. On a longer time-scale shared virtual spaces, with participants represented by avatars, may provide the design rationale for the development of a Learning Surgery in a PET-World.

Asynchronous conferencing systems and email could also be used to conduct personal and small group tutorials. The advantage here might be an emphasis on reflective thinking. These systems could also provide an 'out of hours' emergency service. For example, learners having trouble with assignments could post a request for an urgent synchronous appointment with a PET to discuss the problem. Perhaps a bulletin board system could be used which would allow learners to page a PET by using a special code.

\section{Telementoring}

An example of how access to networked conferencing tools can facilitate mentoring is given by work at Georgia Institute of Technology. In an architecture class, students were asked to use CoWeb (a co-operative Web-based environment) to create two pages for each project they completed: (i) a project description with scanned images of their drawings (pin-ups) and (ii) a statement of research questions to which they needed answers in order to complete their designs. Guzdial (1998) describes how expert architects acted as mentors by using CoWeb:

Expert architects were invited to tour the students' pin-ups and comment on the projects. For each expert architect, a 'tour page' was set up with the architect's name on it. The architect was invited to visit each of the pin-up pages listed on his or her tour, and comment on the pin-ups either directly on the student's page or on the tour page. This activity was perceived as being more successful in supporting the students' learning. The experts wrote a surprising amount of commentary. They sometimes left comments on students' pin-up pages with particular advice, and sometimes they wrote on the tour page with general advice that the expert felt that the group needed. Students took the reviews quite seriously and worked to respond to the experts' comments. (Guzdial, 1998)

\section{Design studio}

The open malleable nature of distributed multimedia provides opportunities for PETs to act as the designers of multimedia educational materials. In this capacity they will need to have access to a range of multimedia authoring tools. Within the Design Studio it is possible to envisage a number of ways of providing access:

- links to locally stored generic tools (e.g. DTP packages, modelling systems, spreadsheets) and authoring packages (e.g. systems such as Authorware Professional, Director, etc.);

- links to Web sites offering shareware design and development tools;

- links to the Web sites of commercial vendors of design/development tools;

- links to collaborative design and development environments.

60 
Resource centre

Learners are going to be confronted with ever increasing amounts of information, and an expectation that their work should be characterized by information-rich exploration and expression. They will need help in selecting information and resources. There is a role here for the PET as an information consultant - someone who will point learners in the direction of useful and relevant resources. In this sense PETs will act like librarians.

At a simple level this support could just take the form of a catalogue of useful resources (Web sites). However, there is a need to customize and personalize support. PETs could give advice on where to search for relevant information and on appropriate search engines to use. Perhaps they could engage in collaborative searching, using shared applications. Intelligent agents may be very useful in the future. Perhaps the possibility of a mixture of PET and intelligent agent support would be a good idea.

One design possibility centres on the concept of mirror virtual worlds which 'provide a second-person experience in which the viewer stands outside the imaginary world, but communicates with characters or objects inside it' (McLellan, 1996, p. 460). A digitized video image of the user is generated and superimposed on the virtual world with its objects and characters, allowing an interaction between the user and the inhabitants of the virtual world. Lewis and Cosier (1997) refer to a mirror system for virtual conferences which mixes real video, avatars, and a pictorial representation of an intelligent agent. In such an environment a PET could project his or her presence into a virtual resource centre visited by avatars representing learners in need of advice about resource and/or information location. A learner's avatar and a PET could communicate to establish the information needed and how best to obtain it. On the basis of these decisions an intelligent agent could be configured to effect the search and deliver the results.

\section{PET-Worlds in higher education}

Is the concept of a PET-World relevant in higher education systems which are being metamorphosed by radically changing attitudes, resource levels and expectations? How would PETs relate to the rapid expansion of higher education, with the implied need to cater for significantly increased student numbers from varied cultural and educational backgrounds? Would PETs help to meet demands for improvements in the quality of university teaching or would they compromise attempts to improve quality? Would there be possibilities for improving cost-effectiveness and better use of resources?

There is clearly a simplistic argument that the adoption of a paradigm based on the extensive use of distributed global networked systems will inevitably lead to improved access, making higher education more available to more people in a variety of locations unbounded by temporal constraints. However, this is a superficial argument which makes a number of assumptions. First, there is an assumption that access to the technical infrastructure supporting PET-Worlds will be widespread, if not universal. While information and communications technologies are becoming pervasive, it is probable that in the foreseeable future some potential students will not have access to appropriate computing facilities. In addition, will all students be able to afford PET fees? Thus the potential to improve access implies significant equity issues. 
What mechanisms and funding issues need to be considered to ensure equitable access? In some ways the establishment of the University for Industry and the National Grid for Learning presage the type of developments that would be necessary to encourage the development of PET-Worlds. Other funding models may introduce equity problems. For example, if PETs become common it is reasonable to assume that there would be an increase in telecommunications traffic resulting in increased revenue. Increased use of infrastructure will result in more demand for hardware and more demand for better connectivity (extent and speed). In this context infrastructure providers may assume a role as a third party broker of educational services, with implications for the price of accessing PET services.

As access becomes more universal and varied in character, conventional institutions may be placed under threat, e.g. through the widespread development of agencies which broker the services of self-employed educationalists. This is very much the model adopted by the widely quoted University of Phoenix. This could lead to a gradual decline in standards. What quality-assurance procedures would need to be established to counter any such decline?

The university curriculum may be compromised, with only those subjects offered by PETs which fit easily into the PET-World framework. The prospectuses of existing virtual universities indicate that this could be a problem, with a very heavy emphasis on vocational subjects such as business studies, nursing and education, to the exclusion of specialized academic subjects such as classics and nuclear physics. Would the widespread adoption of the PET paradigm lead to an expansion in vocational education at the expense of academic scholarship?

The establishment of PET-Worlds implies a large audience for educational materials, which in turn implies a cost-effective approach to resource provision. In particular the arguments rehearsed by Mayes and Fowler (1999) for the reuse of students' materials become very attractive. However, the initial cost of the development of high-quality technology-based learning materials should not be underestimated. These initial costs will need to feature in any financial models for the development of PET-Worlds.

Clearly this discussion is limited and speculative, based as it is on a design specification and limited prototype development. Nevertheless, it points up some important potential advantages and disadvantages of embarking on the developments of PET-Worlds. While there are potential difficulties and pitfalls the PET paradigm could offer much in helping universities to cope with a radically changing higher education context.

\section{References}

Anderson, B. and McGrath, A. (1997), 'Strategies for mutability in virtual environments', Proceedings of Virtual Environments on Internet, WWW, and Networks, National Museum of Photography, Film and Television, Bradford, http://www.visual.bt.co.uk/users/abc/andersb2/public/papers/SfM.doc.

Bradley, L., Walker, G. and McGrath, A. (July 1996), 'Shared spaces', British Telecommunications Engineering Journal, 15, http://vb.labs.bt.com/msss/IBTE_SS/. 
Crossley, M., Davies, N. J., Taylor-Hendry, R. J. and McGrath, A. J. (1997), 'Threedimensional Internet developments', British Telecom Technology Journal, 15 (2), 179-93.

Mayes, J. T. and Fowler, C. J. (1999), 'Learning technology and usability: a framework for understanding courseware', Interacting with Computers, 11 (5), 485-97.

Guzdial, M. (1998), 'Collaborative websites to support an Open Authoring Community on the Web', http://guzdial.cc.gatech.edu/papers/CoWeb/.

Hübscher, R., Puntambekar, S. and Guzdial, M. (1997), 'A scaffolded learning environment supporting learning and design activities', Proceedings of the American Educational Research Association Meeting 1997, http://guzdial.cc.gatech.edu/papers/aera97/scaffolding.html.

Illich, I. D. (1971), Deschooling Society, Harmondsworth: Penguin Books.

Lewis, A. V. and Cosier, G. (1997), 'Whither video? - pictorial culture and telepresence', British Telecom Technology Journal, 15 (4), 64-85.

Martin, D. (1997), 'Putting teachers and parents in control: Internet content labelling and blocking technologies', Proceedings of the National Educational Computing Conference, Seattle, Washington, USA, 281-6.

McFarlane, M. C. (1996), 'Humanizing the superhighway', Technology and Society, 14 (4), 11-18.

Norman, K. (1998), 'Collaborative interactions in support of learning: models, metaphors and management', in Hazemi, R., Hailes, S. and Wilbur, S. (eds.), The Digital University: Reinventing the Academy, London: Springer, 39-53.

Platt, P. and Willard, A. (1998), 'The ramblers guide to virtual environments', IEE Colloquium: The 3D Interface for the Information Worker (May 1998), http://www.hfnet.bt.co.uk/projects/IEE_paper/IEE_paper_v1.htm.

Schlager, M., Fusco, J. and Schank. P. (in press), 'Conceptual cornerstones for an on-line community of education professionals', IEEE Technology and Society, http://www.tappedin.org/info/ieee.html.

Shneiderman, B., Alavi, M., Norman, K. and Borkowski, E. (1995), 'Windows of opportunity in electronic classrooms', Communications of the ACM, 38, 19-24.

Sloffer, S., Duebur, B. and Duffy, T. (1999), 'Using asynchronous conferencing to promote critical thinking: a case study of three implementations in higher education', Proceedings of the Hawaii International Conference on Systems Science, (CD-ROM), Piscataway, NJ: IEEE Computer Society Press.

Squires, D. (1999), 'A teacher's PET for the millennium', in Tuomi-Kyro, E., Salonen, M., Saarinen, P. and Sinko, M. (eds.), Communications and Networking in Education: Learning in a Networked Society Conference Proceedings, Aulanko-Hameenlinna, Finland, 328-33.

Walker, G. (April 1997), 'The mirror - reflections on inhabited TV', British Telecommunications Engineering Journal, 16, 29-38, http://vb.labs.bt.com/msss/IBTE_Mirror/intro.htm. 